\title{
Research the mobile phone operation interfaces for vision-impairment
}

\author{
Yen-Ting $\mathrm{Yao}^{a}$ and Cherng-Yee Leung ${ }^{b}$ \\ ${ }^{\mathrm{a}}$ Graduate Student, Graduate Institute of Design Science, Tatung University, Taipei, Taiwan, Republic of China \\ ${ }^{\mathrm{b}}$ Associate Professor, Department of Industrial Design, Tatung University, Taipei, Taiwan, Republic of China
}

\begin{abstract}
Due to the vision-impaired users commonly having difficulty with mobile-phone function operations and adaption any manufacturer's user interface design, the goals for this research are established for evaluating how to improve for them the function operation convenience and user interfaces of either mobile phones or electronic appliances in the market currently. After applying collecting back 30 effective questionnaires from 30 vision-impairment, the comments have been concluded from this research include: (1) All mobile phone manufactures commonly ignorant of the vision-impairment difficulty with operating mobile phone user interfaces; (2) The vision-impairment preferential with audio alert signals; (3) The visionimpairment incapable of mobile-phone procurement independently unless with assistance from others; (4) Preferential with adding touch-usage interface design by the vision-impairment; in contrast with the least requirement for such functions as braille, enlarging keystroke size and diversifying-function control panel. With exploring the vision-impairment's necessary improvements and obstacles for mobile phone interface operation, this research is established with goals for offering reference possibly applied in electronic appliance design and. Hopefully, the analysis results of this research could be used as data references for designing electronic and high-tech products and promoting more usage convenience for those vision-impaired.
\end{abstract}

Keywords: 3C product, Human Factors, Interface Design, Tactile Sensations

\section{Introduction}

The number of visually impaired people is increasing year by year. The greatest inconvenisnce that plagues visually impaired people is the fact that they are unable to or hardly recognize objects through the visual function. They therefore have to rely on other sensory functions as learning channels to sense the entire external environment. Today, as new electronic and information products being introduced on a constant basis, users are required to learn and understand the functions before they are able to manipulate a product. Because of their inability to receive information via visual senses, visually impaired people experience more learning difficulties than sighted people do. They are surrounded with inconvenisnces in life.

Whenever using mobile phone, users utilize their visual, auditory and tactile senses to receive the information ffrom the mobile phone and quickly convey it to the brain for understanding of the actual operation of the mobile phone. They then press the button on the mobile phone to adjust the mobile phone in order to attain the purpose of using the mobile phone. Visually impaired people lack the ability to receive through the visual sense, which happens to be primary channel design for communication with the mobile phones.

Accompanying with the progress of science and technology, the mobile phones are commonly designed with diversifying functions. Nevertheless, these functions needed, how to operate these functions, or to turn on/off these functions, sight is almost a must.

\section{Literature review}

\subsection{Auditory sense}

The vision-impairment does need vocal-auxiliary control interface or panel whenever operating any electronic alliance. Only the vocal-auxiliary control interface design can (1) Relieve the visionimpairment of obstacles for product usage; (2) Effec- 
tively control the user interfaces or panel; (3) Effective receive and transmit communication and messages in term of vocal signals.

It is commonly mistaken to believe the visionimpairment definitely as being capable of compensating vision communication obstacle by auditory, tactile or sensory ability better than in average. Actually the vision communication obstacle can't be reached effective without vocal auxiliary control design. Such an incorrect belief leads to ignorance of improving more convenient usage and control functions commonly by electronic product manufacturers. Thanks to training and concentration, not enhanced keenness of residual organs, visually impaired people are able to clarify the position and direction of the sound, keep track of the movements and changes of the sound and predict the distance between themselves and the source of the sound in a timely manner.

\subsection{Concept development}

Due to the lack of visual organization ability, visually impaired people hardly identify the object entirely. They can only develop understanding of the parts in order to construct an impression of the whole. They rely on tactile explorations of the hand. The approach, however, limits their ability to describe the depth and entirety of the object in detail.

\subsection{Interface design}

Suitable user interface/control panel design shall be regarded as the most essential for inter-option shifting probability and operational process in emergencies. The control panel design shall feature sufficient keystroke space selecting the exactly required function and preventing pressing down neighboring keystrokes accidentally. The hands by which users operate the remote control must be able to move freely among all the options of the remote control. The remote control must be so designed that no visual sense is required for its operation.

Technology advances have given birth to electronic products of diverse functional types in our daily life and made the operations more complicated than ever. To reduce the operational complexity, some hint functions need to be incorporated in the product interface. The hint functions mentioned here refer to presentations in visual elements such as text, icon and symbol, as well as sound and voice aids for auditory stimulation. To the visually impaired, tactile stimulations like Braille and tactile aids are also required.

\section{Research and purpose}

Through the visual senses, in conjunction with auditory senses or tactile senses, users receive the information and quickly transmit it to the brain, where the information is judged and processed together. Visual defect prevents visually impaired people from receiving visual signals. They can only receive other type of messages. Current design of the mobile phones is based on visual information, yet visually impaired people can only receive mobile phones operation information that is based on the tactile and auditory senses. For them, correct use of the mobile phone is difficult.

Faced with a rapidly-shifting life of technology, human beings for the sake of enhancing product operation and understanding use the graphic user interface (GUI) extensively. For sighted users, icon interface improves product usability. For those visionimpaired users, however, this message transmission method simply doesn't work. They, therefore, are in dire need of a way that would help them acquire information (see Table 1).

As a result of technological progress, home appliance control technology is becoming more significant in the information industry. Yet all the mobil phones operations require the assistance of visual senses. Technological progress has not given visually impaired people more convenience.

The mobile phone is chosen as the analysis target in this research due to its high usage frequency of mobile phones in daily life nowadays. The research methodology was executed by questionnaire of which the major inquiry subjects include (1) the currently common consumer usage status of mobile phones, (2) usual operation preferences, and (3) preferred function and operation improvements for mobiles phones for exploring what obstacles currently the vision impairment suffer arising from mobile phone user operation interface and control panel design that could possibly be applied on improving product design and reference data for researches in future. 
Table 1

People's experience about use the mobile phones with button

\begin{tabular}{|l|l|l|}
\hline Part & Visual experience & Listening experience \\
\hline Screen & $\mathrm{O}$ & $\mathrm{X}$ \\
\hline Operation Button & $\mathrm{O}$ & $\mathrm{X}$ \\
\hline Number Button & $\mathrm{O}$ & $\mathrm{X}$ \\
\hline Speaker & $\mathrm{O}$ & $\mathrm{O}$ \\
\hline Microphone & $\mathrm{O}$ & $\mathrm{X}$ \\
\hline Connection hole & $\mathrm{O}$ & $\mathrm{X}$ \\
\hline Battery & $\mathrm{O}$ & $\mathrm{X}$ \\
\hline
\end{tabular}

\section{Research method and steps}

\subsection{Process}

The experimenter read the questionnaire to participants. In accordance with their experiences with the mobile phone test participants answer the question one by one, and the experimenter recorded their answers on the questionnaire. Each questionnaire interview lasted for about 20 minutes (see Fig 1).

\subsection{Subject}

This research is characterized for 20 interviewees as being vision-impaired (inclusive of 3 suffering Amblyopia and 17 total blindness, respectively); in which each of all interviews own a legal Handicapped Certificate and certain mobile-phone usage experiences; and are aged ranging between 20 and 50 ; including 8 males and 12 females.

\subsection{Data analysis}

The methodology design for the questionnaire has been designed for serving the purposes of integrating and separating all possible mobile-phone usage/operation obstacles into three categories: (1) Frequent usage problems; (2) Operation satisfaction level; (3) Highly-expected improvements for mobilephone function operation design. The answers mechanism for this questionnaire survey has been designed with single-answer choice, listing various combinations of questions for meeting each interviewee's personal preference, and applying a FivePoint Arbitrary Scale and t-Testing for inspecting variances and making statistics; Friedman test was used to assess the order. If the ANOVA showed a significant result, the least significant difference (LSD) method was used to conduct a post hoc test.

\section{Experiment outcome and statistical analysis}

\subsection{Descriptive statistics}

With the aforesaid statistics listed in the table above, it can thus be deduced conclusions stated as below (see Table 2) : (1) mobile usage experiences all average more than 6 years, (2) usage of built-in ring tones is more prevalent, (3) daily carrying the mobile phone constitutes the biggest ratio, (4) seeking assistance for proxy procurement accounts more than $50 \%$ in contrast with extremely low ratio of personal shopping, (5) more than one half unknown the current mobile function/performance status, (6) $70 \%$ users with total numbers in contact list less than 10 in contrast with $25 \%$ having more than 16 numbers in the contact list, (7) an average renewal frequency spanning less than 3 years of mobile phones for $85 \%$ (vision-impairment) users.

\subsection{5-point Likert-scale questions}

The questionnaire methodology wad adopted with Objective Quantitative Analysis featuring a 5-Point Scale, in conjunction with applying the "t-Testing" on validating the significance cause of variances.

From evaluating the aforesaid statistics, it can thus deduce the conclusions as stated as below (see Table $3 \&$ Table 4) : (1) all interviewees commonly satisfactory with current their own phone mobile control panel designs, (2) high ratio of users pretty unacceptable with built-in mobile phone functions, (3) among all preferred suggestions for function improvements, widening keystroke gaps, adding vocal alert, enlarging vertical heights and deepening concaves of keystrokes demonstrating the highest significance, 


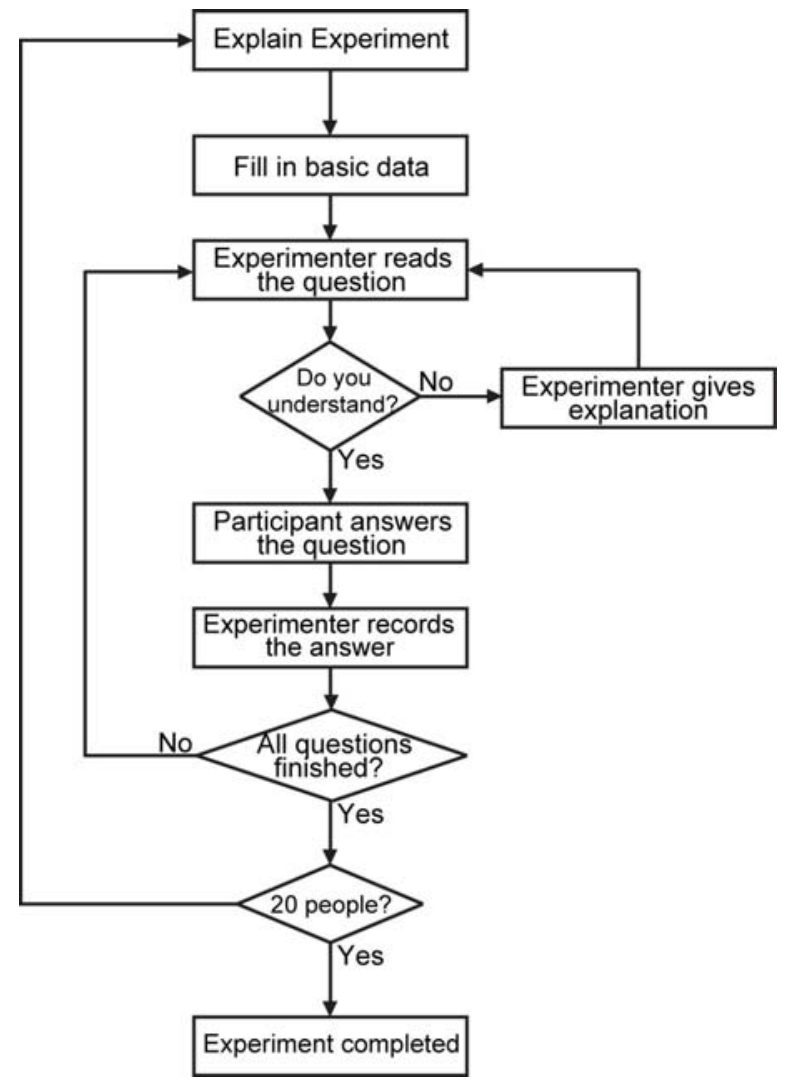

Figure 1. Current use questionnaire survey flowchart

Table 2

Descriptive statistics table

\begin{tabular}{|c|c|c|}
\hline \multirow[t]{2}{*}{ Years of using mobile phones experience } & $6 \sim 10$ years & $15(75 \%)$ \\
\hline & $11 \sim 15$ years & $5(25 \%)$ \\
\hline \multirow[t]{2}{*}{ Selected ring tone } & Built-In & $12(75 \%)$ \\
\hline & Not Built-In & $8(25 \%)$ \\
\hline \multirow[t]{2}{*}{ Usage frequency } & Daily Carry & $19(95 \%)$ \\
\hline & Non-Daily Carry & $1(5 \%)$ \\
\hline \multirow[t]{3}{*}{ Shopping venue } & Mobile Phone Shop & $6(30 \%)$ \\
\hline & Website & $0(0 \%)$ \\
\hline & Proxy Shopping & $14(70 \%)$ \\
\hline \multirow{2}{*}{$\begin{array}{l}\text { Current mobile phone function / perform- } \\
\text { ance obstacles }\end{array}$} & Known & $9(45 \%)$ \\
\hline & Unknown & $11(55 \%)$ \\
\hline \multirow[t]{5}{*}{ Total phone numbers in contact list } & 0 & $5(25 \%)$ \\
\hline & $1 \sim 5$ & $4(20 \%)$ \\
\hline & $6 \sim 10$ & $5(25 \%)$ \\
\hline & $11 \sim 15$ & $1(5 \%)$ \\
\hline & $16 \sim$ & $5(25 \%)$ \\
\hline \multirow[t]{6}{*}{ Mobile phone renewal frequency } & 1 year & $8(40 \%)$ \\
\hline & 2 year & $7(35 \%)$ \\
\hline & 3 year & $2(10 \%)$ \\
\hline & 4 year & $1(5 \%)$ \\
\hline & 6 year & $1(5 \%)$ \\
\hline & 10 year & $1(5 \%)$ \\
\hline
\end{tabular}


Table 3

Current mobile phone usage preferences (t-test: $\mathrm{M}_{0}=3$ )

\begin{tabular}{|l|l|l|l|}
\hline \multicolumn{2}{|c|}{ Current mobile phone usage preferences (t-test: $\mathrm{M}_{0}=3$ ) } \\
\hline & Mean $(\mathrm{Sd})$ & $\mathrm{T}$ & $\mathrm{P}$ \\
\hline Suitable for uncomplicated function demands & $3.95(0.10)$ & 4.25 & $0.00^{*}$ \\
\hline Preference of simple operation & $3.75(1.07)$ & 3.14 & $0.01^{*}$ \\
\hline Easy/clear readier/recognizing keystrokes & $4.35(0.75)$ & 8.10 & $0.00^{*}$ \\
\hline Ergonomic keystroke design & $4.25(0.64)$ & 8.75 & $0.00^{*}$ \\
\hline Durable keystroke manufacturing materials & $3.80(0.77)$ & 4.66 & $0.00^{*}$ \\
\hline $\begin{array}{l}\text { Sufficient vocal alert function after pressing } \\
\text { each function keystroke }\end{array}$ & $4.65(0.67)$ & 11.00 & $0.00^{*}$ \\
\hline Message communication & $2.45(1.39)$ & -1.76 & 0.09 \\
\hline
\end{tabular}

Table 4

Functions required for any improvement project (t-test: $\mathrm{M} 0=3$ )

\begin{tabular}{|l|l|l|l|}
\hline & Mean $(\mathrm{Sd})$ & $\mathrm{T}$ & $\mathrm{P}$ \\
\hline Adding the function of braille & $3.00(1.52)$ & 0.00 & 1.00 \\
\hline Widening space gaps among all panel keystroke & $4.00(0.86)$ & 5.21 & $0.00^{*}$ \\
\hline Enlarging voice output volume & $4.45(1.28)$ & 5.08 & $0.00^{*}$ \\
\hline Enlarging keystrokes & $2.70(1.22)$ & -1.10 & 0.29 \\
\hline Multiple vibration modes & $3.05(1.10)$ & 0.20 & 0.84 \\
\hline Multiple keystroke shapes & $2.90(1.33)$ & -0.34 & 0.74 \\
\hline $\begin{array}{l}\text { Enlarging vertical heights and deepening con- } \\
\text { caves of keystrokes }\end{array}$ & $3.55(1.05)$ & 2.34 & $0.03^{*}$ \\
\hline
\end{tabular}

(4) nearly no significance of preference in terms of adding braille, enlarging keystrokes, multiple vibration modes, multiple keystroke shapes.

From evaluating the aforesaid statistics, it can thus deduce the conclusions as stated as below (see Table 3 \& Table 4) : (1) all interviewees commonly satisfactory with current their own phone mobile control panel designs, (2) high ratio of users pretty unacceptable with built-in mobile phone functions, (3) among all preferred suggestions for function improvements, widening keystroke gaps, adding vocal alert, enlarging vertical heights and deepening concaves of keystrokes demonstrating the highest significance, (4) nearly no significance of preference in terms of adding braille, enlarging keystrokes, multiple vibration modes, multiple keystroke shapes.

\subsection{Ranking and clustering}

The questionnaire for this research has been designed with multiple combinations of questions for allowing the interviewees to make answers in se- quence according to personal preferences with applying the Friedman Testing on analyzing each interviewee's answers afterwards and making statistics processing for overall survey results.

The aforesaid evaluation statistics thus can be used for deducing the conclusions stated as below (see Table 5 \& Table 6) : (1) Most preferential with the built-in audio speech/informer When considering buying a mobile phone, with regarding the interior design and extra functions as the least important when making consideration for mobile phone shopping, (2) Vision-impairment incompetent of learning mobile phone function operation methods unless independently without assistance from others, (3) A prevalence for most vision-impairment users simply switching off and restarting the mobile phone in case of failing to operate as required, (4) Answering and making a phone call constituting the most usage frequency with the lowest frequency of using gameplaying and personal calendar and event reminder.

Table 5

Evaluation factors for mobile-phone procurement among vision-impaired users (Friedman test / LSD Layout Ranking)

\begin{tabular}{|l|l|}
\hline$\chi_{0}^{2}$ & 106.5 \\
\hline P-value & $0.00<0.05$ \\
\hline \multirow{4}{*}{ Overall preference (LSD grouping) } & Built-in message speech \\
\cline { 2 - 2 } & Ergonomic keystroke design for easy recognition prior to pressing the keystroke as required \\
\cline { 2 - 2 } & Built-in vibration alarm and informer \\
\cline { 2 - 2 } & Plenty of built-in functions with pleasant exterior design \\
\hline
\end{tabular}


Table 6

ETroubleshooting in case of usage obstacles (Friedman test / LSD Layout Ranking)

\begin{tabular}{|l|l|}
\hline$\chi_{0}{ }^{2}$ & 5.7 \\
\hline P-value & $0.13<0.05$ \\
\hline \multirow{2}{*}{ Overall preference (LSD grouping) } & Press the recovery key for back to main function display and switching off the mobile phone \\
\cline { 2 - 2 } & $\begin{array}{l}\text { Switching off } \\
\text { Seeking assistance from others }\end{array}$ \\
\hline
\end{tabular}

\section{Conclusions}

With applying statistical methodology on the results collected from the questionnaire survey, it can thus validate for this research the analysis results stated as below:

- The vision-impairments commonly feature a high frequency of mobile-phone renewal, of which most of them preferential with mobilephone renewal once in less than 3 years. Such an analysis result can validate for most of them the mobile-phone usage as necessary, and easy learning for function operation as pretty important.

- The vision-impairments demonstrate a confirmation concerning all mobile-phone designs currently with a significance (inclusive of meeting personal usage requirements, simple and easy function operations, audio message and communication alert/informer, function access by pressing keystrokes and exterior design) . The vision-impairments still suffer difficulty in function operation and usage of all mobile phones manufactured with various design concepts in the market currently.

- $70 \%$ vision-impaired users have less than 10 phone numbers saved in contact list with contrast $25 \%$ users having more than 16 with a possibility that adding the audio-signal alert can explain the latter having more phone numbers recorded in the contact list. Meanwhile, those vision-impaired users without equipping audio signal alert function could simply use the quick- dial application as an alternative for usage assistance.

- Low ratio of the vision-impaired users character deciding mobile-phone procurement independently, being incompetent of reading mobilephone manual guide, with necessity of seeking assistance from others in procurement and instruction for function operation; possibly the vision-impairment causing the necessity for seeking due to being incompetence of making judgment independently, the necessity of seeking other people for assistance in sharing usage experiences and teaching function operations.

- Among all mobile-phone functions, generating an audio signal upon pressing any keystroke is regarded as the most favorable and convenient. Likewise, adding an audio speech function is also deemed as highly necessary. All those can justify integrating the audio signal function into mobile-phone user interface design.

- Among most vision-impaired users, there exists a highly prevalent phenomenon simply switching off and restarting the mobile phones whenever failing to reach troubleshooting against operation obstacles. It thus can justify that the mobile-phones featuring a design concept of a recovery key function would definitely prevent operation discomfort significantly and reduce incorrect operation probability and offer more operation convenience for the vision-impaired users.

Table 7

The prevalent method for learning how to operate a newly-bought mobile phone by the vision-impairment (Friedman test / LSD Layout Ranking)

\begin{tabular}{|l|l|}
\hline$\chi_{0}{ }^{2}$ & 27.3 \\
\hline P-value & $0.00<0.05$ \\
\hline \multirow{3}{*}{ Overall preference (LSD grouping) } & By inquiring people having similar usage experiences \\
\cline { 2 - 2 } & Learning function operation methods by oneself gradually \\
\cline { 2 - 2 } & Reading product manual guide \\
\hline
\end{tabular}


Table 8

Usage frequency for each of all mobile-phone functions (Friedman test / LSD Layout Ranking)

\begin{tabular}{|l|l|}
\hline$\chi_{0}{ }^{2}$ & 5.7 \\
\hline P-value & $0.13<0.05$ \\
\hline \multirow{5}{*}{ Overall preference (LSD grouping) } & Answering the incoming calling \\
\cline { 2 - 2 } & Switching on/off the mobile phone \\
\cline { 2 - 2 } & Checking time \\
\cline { 2 - 2 } & Alarm function \\
& Audio Walkman \\
\cline { 2 - 2 } & Audio Walkman \\
& Message transmission \\
\cline { 2 - 2 } & Game playing \\
& Usage of built-in personal calendar / event reminder \\
\hline
\end{tabular}

- It can deduce from the research results that the highly expected function improvements including widening keystroke gaps, adding audio signal alertness, enlarging vertical height and deepening concave for every keystroke in contrast with suggestions containing the least or nearly non-existent demand for such as keystroke size, exterior shape, dimension, vibration modes and even equipping with braille function. All those can indicate that, in addition to the demand for audio signal alertness, for the vision-impaired users, another important function shall promoting tactile recognition and response against pressing keystrokes tactile by means of widening keystroke gaps as much as possible as each keystroke is really pretty small.

\section{References}

[1] J. H. Burgess, Human Factors in Industrial Design: the Designer Companion. TPR, Blue Ridge Summit, PA,1989

[2] C. Y. Leung, and W. N. Huang, A Study on the Pattern and Color Contrast of Chinese Characters for Air Condition Remote Control. In: The 4th Academic Research Conference, The Chinese Design Association, Tatung University, Taipei, Taiwan, R.O.C., 1999, pp. 367-370.

[3] C. Y. Leung, Y. T. Yao, and S. C. Chuang, A Study on Requirements and Opinions in Operating Air-condition for Visually Impaired People. In: International Conference on Speciation Education and Art Therapy, Taichung University, Taichung, Taiwan, R.O.C., 2008.

[4] B. Lowenfeld, The Visually Handicapped, Review of Educational Research, Education of Exceptional Children, 33 (1), 1963, pp. 38-47.

[5] M. Shinohara, Y. Shimizu, and A. Mochizuki, ThreeDimension Tactile Display for the Blind, IEEE Transaction on Rehabilitation Engineering, 6 (3), 1998, pp. 249-256.

[6] C. Y. Wu, A Study on Designing the Optimal Parameters of Hierarchical Menu System on 3C Products for Visually Impaired People. Thesis for Master of Science, Department of Industrial Design, Tatung University, Taiwan, 2006. 